\title{
Functionality and Performance of the Subretinal Implant Chip Alpha AMS
}

\author{
Renate Daschner, ${ }^{1}$ Albrecht Rothermel, ${ }^{2}$ Ralf Rudorf, ${ }^{1}$ \\ Sandra Rudorf, ${ }^{1}$ and Alfred Stett ${ }^{1 *}$ \\ ${ }^{1}$ Retina Implant AG, Gerhard-Kindler-Strasse 13, 72770 Reutlingen, Germany \\ ${ }^{2}$ Institute of Microelectronics, University of Ulm, Albert-Einstein-Allee 43, 89081 Ulm, Germany
}

(Received August 18, 2017; accepted November 8, 2017)

Keywords: retinal prosthesis, retinitis pigmentosa, subretinal stimulation, clinical device reliability

Electronic retinal implants have been developed and are marketed as a therapeutic option for blind people suffering from degenerative retinal diseases such as retinitis pigmentosa. The functionality of subretinal implants depends heavily on the performance of the electronic interface to the retina. For the RETINA IMPLANT Alpha AMS device, this interface consists of a subretinally implanted chip that samples the retinal image, like a camera chip, and stimulates the adjacent retina simultaneously at the corresponding locations. The technical functionality of the RETINA IMPLANT Alpha AMS is described and compared with the outcome of two clinical trials over an observation period of one year. The discrimination of different grey levels observed in these clinical trials confirms that the sensitivity of the implanted CMOS chip can be varied over the range of relevant light intensities. We show that accelerated aging lifetime measurements of implant components in a laboratory environment match implant lifetimes observed during clinical trials for the predecessor device, the RETINA IMPLANT Alpha IMS. By using the same model for the current technically advanced device, the RETINA IMPLANT Alpha AMS, the predicted clinical lifetime of the implant is about 5 years.

\section{Introduction}

Retinitis pigmentosa (RP) is a hereditary retinal disease that begins with peripheral visual field defects and can finally lead to complete blindness. It is generally assumed that about one person in every 4000 is afflicted by RP, although for many of these people, the disease does not result in a complete loss of vision during their lifetime. So far, there is no successful therapy that can stop the process of degeneration of the visual cells, which progresses over a period of decades.

After many years of research and development, electronic retinal implants gained market approval in various countries and are now commercially available as a therapeutic option that generates visual perceptions through electrical stimulation of the degenerated retina.

*Corresponding author: e-mail: alfred.stett@retina-implant.de http://dx.doi.org/10.18494/SAM.2018.1726 
Currently, there are three market-approved products that follow different approaches: the subretinal implant RETINA IMPLANT Alpha AMS of Retina Implant AG (hereafter, Alpha AMS; Germany, CE marking 2016), and the epiretinal implants Argus ${ }^{\circledR}$ II from Second Sight Medical Products (USA, CE marking 2011, FDA-PMA 2014) and IRIS ${ }^{\circledR}$ II from Pixium Vision (France, CE marking 2016). An overview of the results obtained by patients using retinal implants has been described by Chuang et al. ${ }^{(1)}$

The quality of vision that can be achieved with retinal implants depends on many circumstances. In addition to patient-specific factors, such as the individual course and status of the retinal degeneration, the electronic interface between the implant and the retina, and the neuronal activation achievable with it, are of particular importance. Hence, in this article, we describe the functional properties of the light-sensitive chip of the subretinal implant Alpha AMS, which establishes an interface to the retina.

As the inner retinal cells are maintained after the loss of photoreceptors, subretinal implants can electrically stimulate the remaining cellular network of the retina. This approach to retinal prostheses assumes that the inner retina of blind patients is still capable of processing information. It is further assumed that the inner retina can be electrically stimulated in such a way that the network-mediated modulation of the ganglion cell activity yields meaningful visual perception.

The primary targets for subretinal electrical stimulation are the voltage-gated $\mathrm{Ca}^{2+}$ channels located at the presynaptic axon terminals (PSTs) of the bipolar cells. The fundamental task is to modulate the membrane voltage at the PSTs to modulate the release of neurotransmitters, and ultimately, the signaling to the amacrine and ganglion cells. Therefore, electrode configurations and stimulation protocols that enable the efficient stimulation of the bipolar cell axons with high spatial resolution simultaneously at multiple locations are required.

The most efficient way to stimulate the extended, cable-like bipolar cells is the application of electric fields, which are directed parallel to the axons of the bipolar cells. It has been shown that a positive current (which is related to the electric field) flowing from the outer retina to the inner retina can depolarize the axon terminals. ${ }^{(2)}$ Ex vivo experiments have verified the stimulating effect on the retina of inward-directed currents. ${ }^{(3,4)}$ The phosphene perceptions evoked by subretinal implants may be attributed to the depolarization of the PSTs by transretinal currents.

With the Alpha AMS (Fig. 1) the stimulation current is delivered by its key component, the light-sensitive CMOS chip (Fig. 2), which is subretinally implanted between the retina and the pigment epithelium. It is equipped with 1600 electrodes in a monopolar configuration. They are embedded into the surface of the chip and are in direct contact with the retinal tissue. Therefore, current generated by the device and injected via the electrodes completely crosses the retina in a perpendicular direction and spreads to the remote return electrode (Fig. 3).

\section{Materials and Methods}

\subsection{General description of the Alpha AMS}

The RETINA IMPLANT Alpha consists of two device families: the RETINA IMPLANT Alpha IMS (hereafter, Alpha IMS) and the Alpha AMS. The Alpha IMS was implanted during 


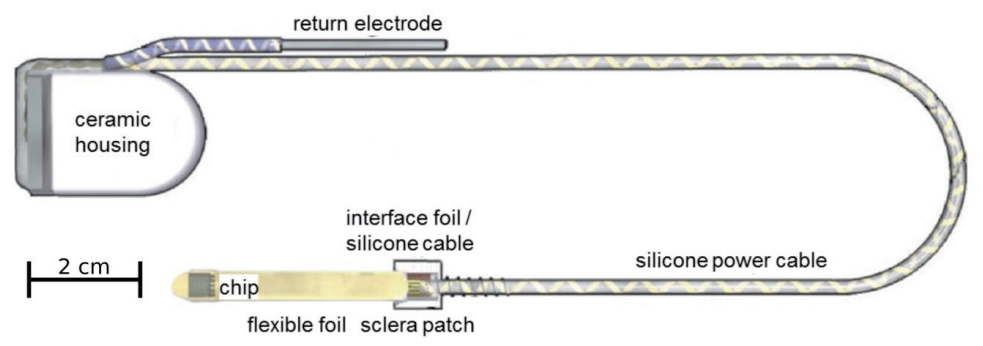

Fig. 1. (Color online) Subretinal implant Alpha AMS. The light-sensitive CMOS chip, mounted on a flexible foil, establishes the interface to the retina, while power is supplied via an inductive link to hermetically housed electronics in a subdermal ceramic housing. The silicone power cable is routed on top of the skull bone (below the periost) and connects the intraocular chip with the electronics in the ceramic housing, which is implanted behind the ear.

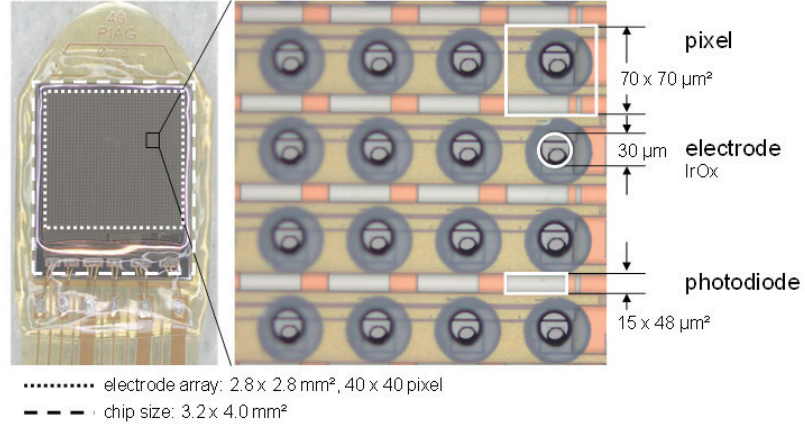

Fig. 2. (Color online) Light-sensitive chip of the subretinal implant Alpha AMS. The electrode array $\left(2.8 \times 2.8 \mathrm{~mm}^{2}\right)$ of the chip contains $160070 \times 70 \mu \mathrm{m}^{2}$ pixels.

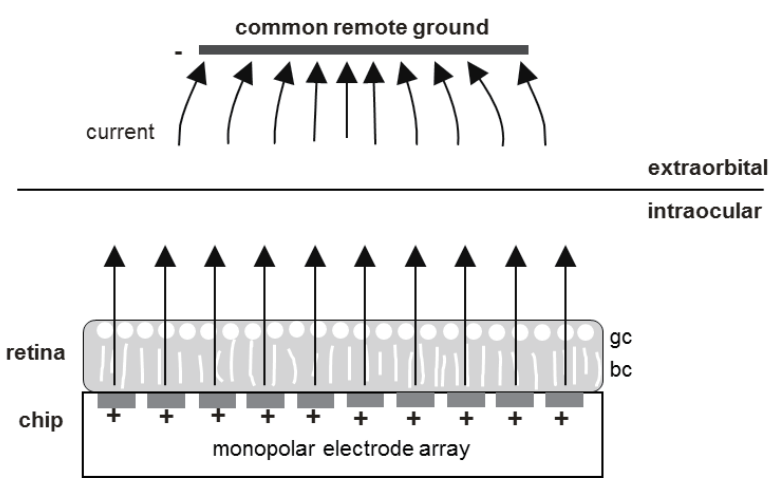

Fig. 3. Schematic representation of the monopolar electrode array for subretinal stimulation. The current (indicated by arrows) spreads transretinally from subretinal stimulating electrodes (marked with $+)$ to the remote return electrode in the extraorbital space (-). (gc: ganglion cell; bc: bipolar cell).

clinical trials in Europe, Hong Kong, and Singapore from May 2010 to September 2013 and its distribution is now discontinued. The Alpha AMS has been used in clinical trials in Europe from February 2014 to the present, received the CE marking in March 2016, and is the currently marketed product.

The surgical procedure for subretinal implantation and the functional outcome with the current device, the Alpha AMS, and its predecessor, the Alpha IMS, have been described. ${ }^{(5-9)}$

The Alpha AMS consists of several components (Fig. 1). In contrast to epiretinal implants, the intraocular part of the subretinal Alpha AMS implant comprises an active electronic chip including photodiodes to sense the light that enters through the natural pathway of the eye. This has the advantages that a large number of electrodes can be addressed, no external camera is needed, and natural eye movements can be used by the patients to locate and fixate on objects. The light that falls on the chip is used to directly control the stimulation current amplitude in each pixel.

The chip is mounted on a thin polymer substrate for trans-scleral power supply and the transmission of control signals. On the outside of the eyeball, the polymer substrate is connected via a power cable to an implanted hermetically encapsulated circuit board. This 
circuit is used to inductively couple an external device with the retinal chip by transforming the incoming modulated RF signal into power and control signals for the chip.

The external device is used to adjust the control signals of the chip. Stimulation frequency and pulse duration can be set via control software, while gain and sensitivity can be controlled by the patient himself to adapt the stimulation to the actual light conditions. The most crucial part of such a retinal implant is the active CMOS chip. Its design has to be adapted to the special conditions in the human eye so that the output signal matches interpretable signals for the neural cells in the eye, imitating the degenerated photoreceptors. A second challenge in the design of an implant is the durability of the CMOS chip materials in the harsh, corrosive environment of the human body.

In the following sections, two chips are compared. The functionality of the CMOS chip of the Alpha AMS implant is described in detail, and the results of the chip reliability are compared with those of the chip of the Alpha IMS implant. The main differences between the two implant versions are changes in the design and the material. They have been summarized by Stingl et al. in 2017. ${ }^{(7)}$

\subsection{Alpha AMS chip functionality and characteristics}

\subsubsection{Electrical functionality of the CMOS chip}

The retina is stimulated by a CMOS chip. It includes an array of $40 \times 40$ pixels, analog and digital control circuitry, and peripheral devices for interconnection. ${ }^{(10)}$ Each pixel cell has dimensions of $70 \times 70 \mu \mathrm{m}$ and includes a photodiode, an amplifier, biphasic current drivers, and a circular stimulation electrode (diameter $30 \mu \mathrm{m}$ ). The electrode material is reactively sputtered Iridium Oxide (SIROF), which is a highly recommended material for stimulation electrodes. ${ }^{(11)}$ Safe charge injection limits of IrOx up to $4 \mathrm{mC} / \mathrm{cm}^{2}$ have been reported. ${ }^{(12)}$

All terminals run DC-free signals, and also the supply voltage of the chip is a biphasic square wave. Optimized electrode drivers that allow for rail-to-rail output voltages are used, which means that no point of the subretinal electronics has to exceed the maximum required electrode potentials. The electrode drivers also deliver biphasic output currents and voltages to optimize charge transfer and the lifetime. The negative and positive peak currents of the drivers are controlled by the photosensors individually in each pixel. Hence, each pixel stimulates the overlying retinal tissue with a current according to the local light intensity. A continuous series of voltage-limited biphasic current pulses is applied to all electrodes synchronously. The sensitivity of the photodiode and the maximum stimulation current of the chip can be adjusted by the patient during normal operation of the implant to suit the ambient light intensity and individual retinal response behavior. The stimulation pulses have a variable repetition frequency and negative and positive phase durations (max. $2 \mathrm{~ms}$ per phase), which can also be adjusted by the control signals.

\subsubsection{Light sensitivity and charge transfer characteristics}

The photosensors are designed with a logarithmic characteristic for the highest dynamic range. Their output signals are amplified and adjusted according to the sensitivity setting of 
the device. The output of the chip can be represented by the characteristic charge transfer curve as a function of the illuminance (Fig. 4). For the measurement of the transfer characteristic, the chip is illuminated by light with eight different intensities from 0.06 to 10000 lx. For each illuminance, several combinations of gain and sensitivity are set at a handheld device. At each setting, the stimulation signal of the chip is measured. The charge released per pixel and per pulse is calculated from the current signal and plotted versus the respective illuminance. This characteristic charge transfer curve can be used to determine the optimum setting of gain and sensitivity for different light conditions. However, the actual electrically evoked light perception for the patient also depends on the individual excitability of the retina.

\subsubsection{Theoretical limit of visual acuity}

The theoretical visual acuity that can be achieved with the chip can be estimated from the distance between neighboring pixels. The minimum angle of resolution (MAR) that is possible with a retinal chip can be calculated using a reduced eye model, ${ }^{(13)}$ which replaces the complex system of several refractive surfaces in the eye with a single nodal point and a focal length of $17.2 \mathrm{~mm}$. The MAR $\theta$ can thus be calculated as

$$
\tan \theta=\frac{\text { pixel pitch }}{\text { focal length }}
$$

According to Eq. (1), the calculated visual angle between two neighboring pixels on top of the Alpha AMS chip is determined to be $0.233^{\circ}$, equivalent to a MAR of 14 min of arc and $\log M A R=1.15$. The visual acuity that can theoretically be achieved with the pixel pitch of 70 $\mu \mathrm{m}$, which is simply the reciprocal of the MAR, is 0.07 , corresponding to a Snellen fraction of 20/280.

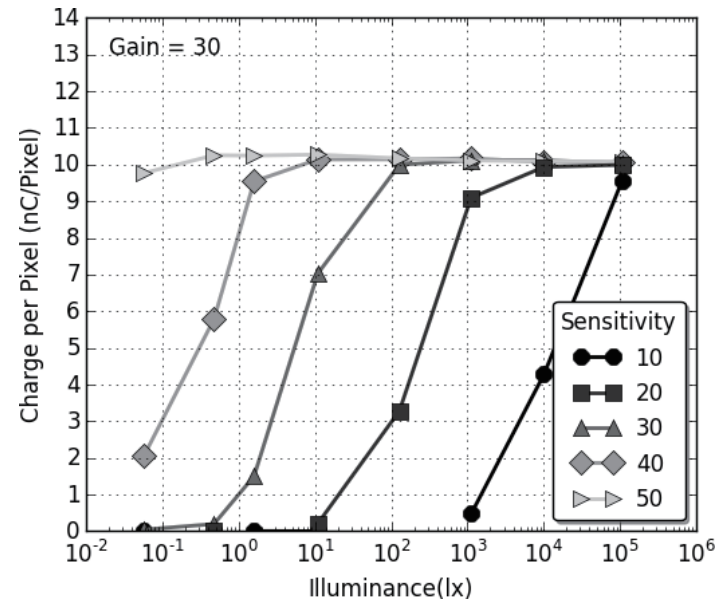

(a)

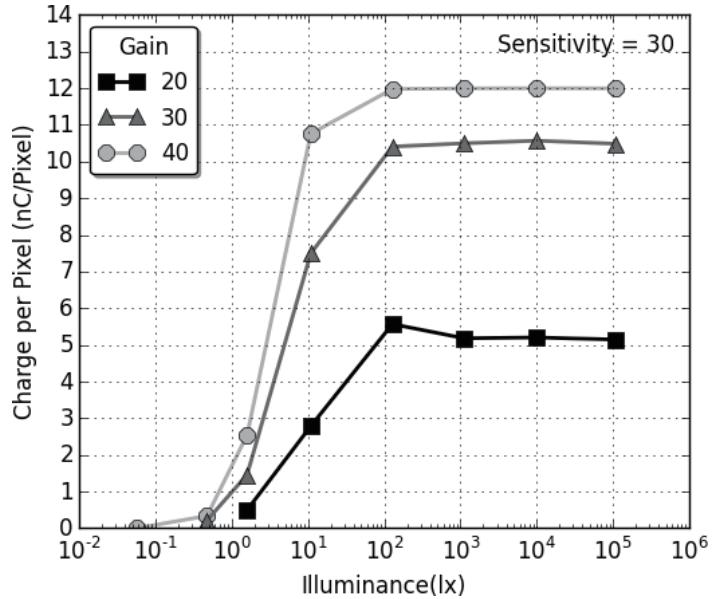

(b)

Fig. 4. Characteristic charge transfer curve of the retinal implant Alpha AMS. Only $50 \%$ of the pixels are activated to simulate realistic clinical conditions. (a) Gain $30 \%$, sensitivity 10 to $50 \%$, (b) gain 10 to $60 \%$, sensitivity $30 \%$. The light sensitivity can be adjusted between $10^{-1}$ and $10^{4} \mathrm{~lx}$. The amplitude of the stimulation charge varies with increasing gain. 
For the detection of a spatial contrast grating with the chip, two successive maxima of the grating must be detected with two rows or columns of pixels separated by at least one row or column, respectively. Thus, the maximum grid resolution (grating acuity) is determined by twice the minimum period length of the grating. The theoretical maximum grating acuity of the retinal chip Alpha AMS is $2.14 \mathrm{cpd}$.

The visual field depends on the size of the electrode area. As a result of the implantation procedure, the chip is usually tilted by approximately $45^{\circ}$ in the eye, so the diagonal length of the chip has to be taken into account to calculate the maximum horizontal visual field. The size of the electrode area of the Alpha AMS chip is $2.8 \times 2.8 \mathrm{~mm}^{2}$, corresponding to a visual field of $9.3^{\circ}$. Owing to the tilt of the chip, the maximum horizontal visual field is increased to $13^{\circ}$.

\subsubsection{Fabrication and encapsulation of the chip}

The CMOS chip is fabricated by ams AG, Austria. Additional processing to improve durability is required to avoid corrosion of the CMOS chip in the humid environment of the body. Hermetic encapsulation of the electronically active chip is not possible for three reasons: First, for a subretinal implant without an external video camera, the image is detected with photodiodes directly on top of the implanted CMOS chip. Thus, a smooth transparent surface above the photodiodes is needed to allow the detection of incoming light. Second, the electrodes of each of the 1600 pixels have to be in direct contact with the adjacent retinal tissue for low-threshold stimulation. Third, for subretinal implantation, the chip has to be as thin as possible. Owing to these requirements, only conformal coatings are possible to protect the chip from corrosion. Thus, the lifetime of the chip mainly depends on the insulating properties of the conformal coating with its 1600 vertical feedthroughs for the electrodes.

\subsection{Reliability of the subretinal chip}

\subsubsection{Laboratory accelerated aging measurements}

To estimate the time that a device will operate, methods of reliability analysis are used. These methods are typically applied in engineering to estimate the lifetime of products. How long a device is working can be described by the reliability function, showing which percentage of devices is expected to function after a specific amount of time. ${ }^{(14)}$ Details about the estimations of the lifetime of the subretinal implants and its components were reported by Daschner et al. ${ }^{(15)}$

The reliability of the implant has been tested in a laboratory environment by subjecting the single components of the implant to the following conditions. The polymer substrate has been tested under accelerated aging conditions at $60{ }^{\circ} \mathrm{C}$, suggesting a median lifetime of 12.7 years. Bending tests with the power cable led to an expected lifetime of 7.0 years. ${ }^{(15)}$ In the next sections, the laboratory tests of the retinal chip, which is the most important and critical component of the subretinal implant, are described in more detail.

Several chips were operated continuously under accelerated aging conditions until chip 
failure occurred. The time to failure was recorded and the failure modes were analyzed. A chip was categorized as failed as soon as a stimulation signal was no longer measurable.

The individually measured lifetimes of the chips were statistically evaluated by a technique widely used in engineering for reliability analysis. Each of the measured times to failure was assigned a median rank, which describes the percentage of chips that have failed. Plotting the corresponding median ranks versus the times to failure results in a reliability distribution describing the failure mechanisms of the system (Fig. 5). By fitting this curve with a Weibull distribution, the median lifetime of the measurements was determined. The median value then represents the time when half of the chips have failed.

The chips were operated with the same handheld device as that used by the patients to guarantee that the laboratory tests are as close as possible to normal device usage. Stimulation parameters that are typical in clinical usage were chosen for the long-term test. The supply voltage was $1.2 \mathrm{~V}$ with a supply frequency of $100 \mathrm{~Hz}$. A pulse width of $2 \mathrm{~ms}$ for each pulse phase and a stimulation frequency of $5 \mathrm{~Hz}$ were used because these are typical values in clinical applications, which in most cases delivers a sufficiently high refresh rate of the image without leading to fading. As the chip was expected to age faster with higher gain and sensitivity, we used the maximum gain and sensitivity to avoid overestimation of the lifetime of the chip.

For a more detailed analysis of the aging effects, the chip was tested separately without connection to other implant parts. In this case, the chip was mounted on a circuit board and encapsulated in the same way as during implant fabrication to enable us to observe the failure modes of only the chip itself. An aging temperature of $60{ }^{\circ} \mathrm{C}$ was chosen to guarantee that no additional aging processes were present as might be the case for higher temperatures. As an approximation for the acceleration factor, the ten-degree rule can be used because of the moderate acceleration temperature of $60^{\circ} \mathrm{C} .{ }^{(16)}$ It assumes that the reaction rate doubles when the temperature is increased by ten degrees. Under the laboratory test condition at $60{ }^{\circ} \mathrm{C}$, the chips age about 5 times faster than under the conditions of use at $37{ }^{\circ} \mathrm{C}$ in patients.

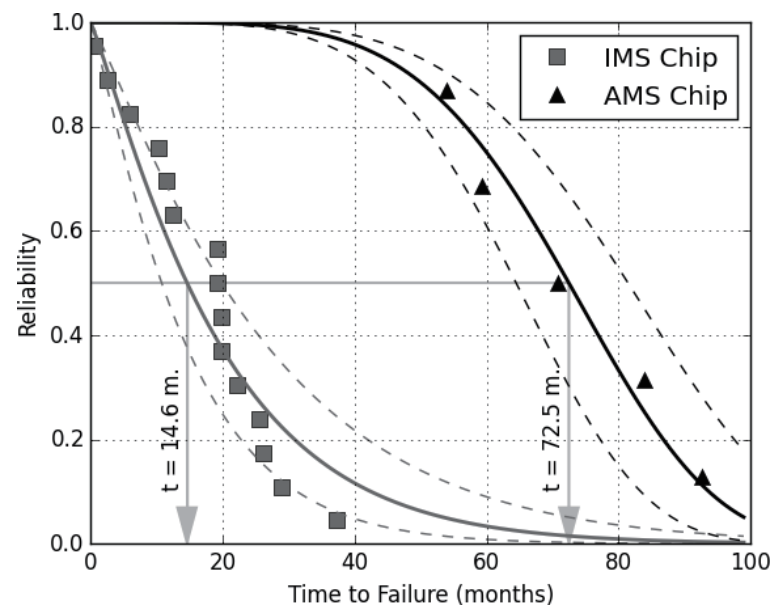

Fig. 5. Laboratory accelerated aging tests of the Alpha IMS chip and the Alpha AMS chip. Reliability is a measure of the percentage of chips that are expected to still function after a specific amount of time. Both datasets are fitted with a Weibull distribution. The dashed lines denote the $75 \%$ confidence level. 


\subsubsection{Clinical device reliability}

The reliability of medical devices is usually described by a Kaplan-Meier plot. $^{(17)}$ The advantage of this method is that it can be used without knowledge of the underlying probability distribution even when some of the devices are still working, as is the case for the Alpha AMS implants in clinical use. The survival rate for each time step is calculated separately and then the cumulative survival rate is determined by multiplication of all previous survival rates. A detailed description of the calculation can be found in the standard ISO 5841-2:2014.

An implanted device is considered as failed when the patient no longer has any visual perception and the failure of the device is confirmed by electroretinography measurements. ${ }^{(18)}$ We use two failure categories to distinguish between purely technical failures of the implant itself (denoted as failure category $\mathrm{C} 1$ ) and other failure mechanisms including procedural errors (denoted as category $\mathrm{C} 2,{ }^{(15)}$ Fig. 6).

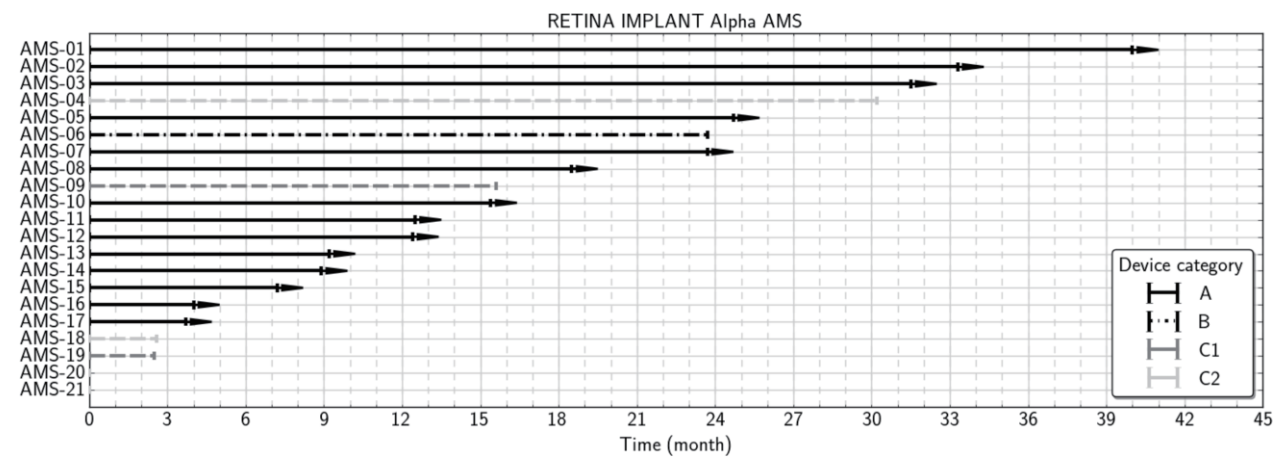

(a)

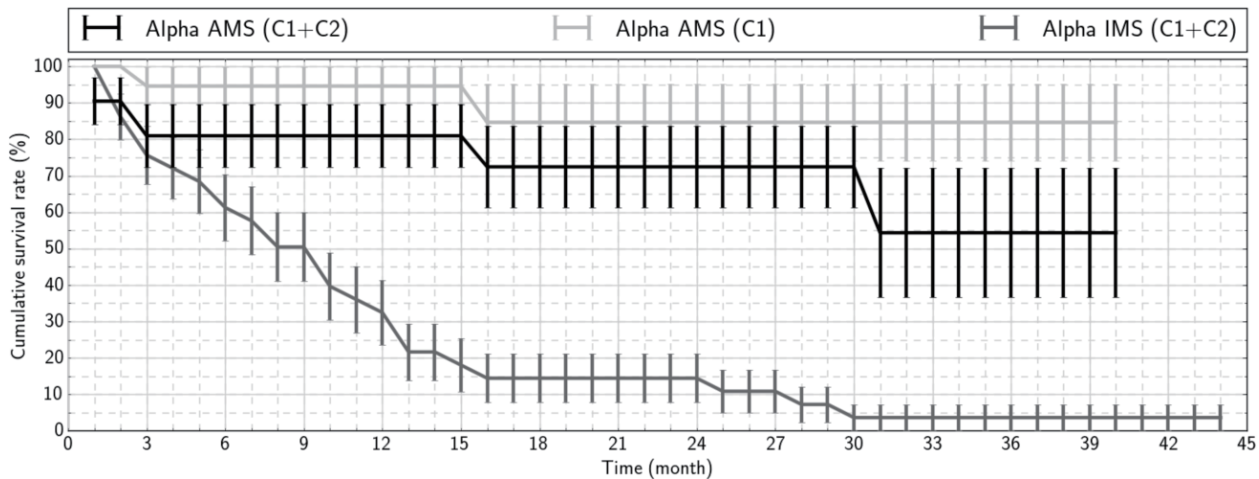

(b)

Fig. 6. (a) Runtime of all Alpha AMS implants in clinical use until 06/30/2017. Category "A" denotes implants that are functioning; category "B" denotes implants that are explanted due to reasons not related to the functioning of the device; categories " $\mathrm{Cl}$ " and "C2" denote implants that are no longer functioning due to technical reasons ("Cl") or other (e.g., procedural) reasons ("C2"). 14 out of 21 implants are still functioning. 12 implants were implanted for more than one year, of which 9 implants are still functioning. (b) Kaplan-Meier plot of the retinal implants Alpha IMS (dark grey) and Alpha AMS (black and light grey). In the light grey curve, only technical failures are included (C1: number of technical failures), while in the black curve, all devices that are no longer in use are included regardless of the failure mechanism $(\mathrm{C} 2$ : number of failure mechanisms that are not due to technical failure). 


\subsubsection{Comparison between clinical and laboratory reliability}

For direct comparison of the clinical lifetime and the laboratory measurements, the time to failure of the retina implants that failed in clinical use was additionally evaluated with the same model as that used for the laboratory experiments. Since the complete data set of Alpha IMS failures in clinical use follows an exponential distribution, an exponential distribution was also used to fit the clinical reliability of the Alpha AMS (Fig. 7).

\subsection{Clinical performance}

The Alpha AMS has been implanted in 16 patients in two clinical trials between 2014 and 2017. Study details and interim functional outcomes of the first 15 patients were described by Stingl et al. ${ }^{(7)}$ All of these patients had end-stage hereditary retinal degeneration with impaired light localization or worse. Fourteen of the patients had RP and one had cone-rod dystrophy. All devices were implanted in one eye at four study sites in Germany and Great Britain (www. clinicaltrials.gov-IDs: NCT01024803 and NCT02720640). The follow-up duration was 12 months, but the implant is left in the eye as long as it provides benefit to the patient. The functional outcomes of the clinical trials were tested by screen-based standardized two- or four-alternative forced-choice tests of light perception, light localization, Landolt C-rings, grey level discrimination, and performance during activities of daily living. The detection of the orientation of gratings of different spatial frequencies was carried out via the basic grating acuity (BaGA) test ${ }^{(19)}$ in which the participants were asked to tell the orientation of a grid of white bars at a range of spatial distances.

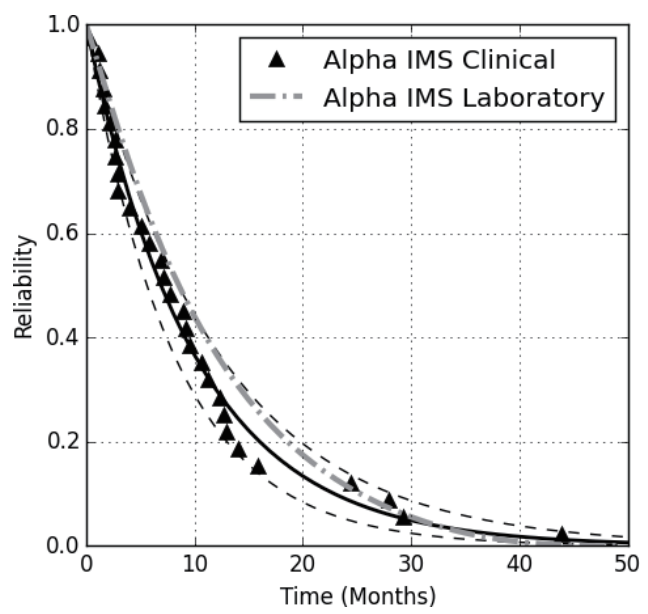

(a)

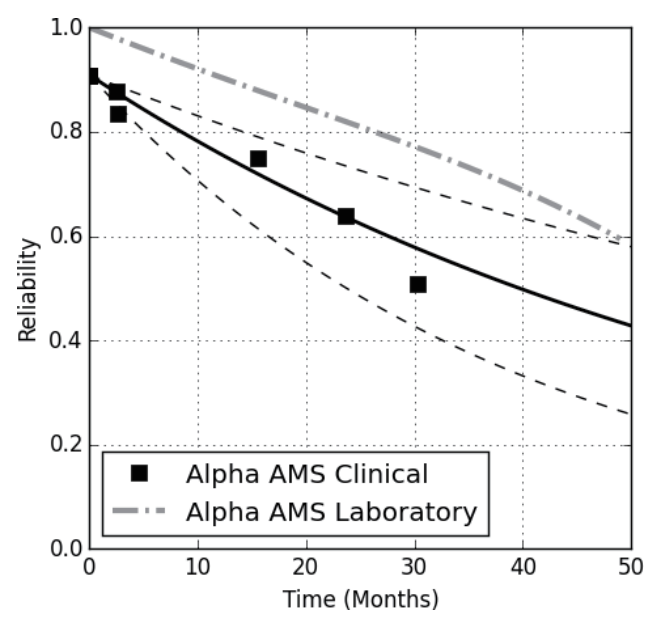

(b)

Fig. 7. Reliability of Alpha IMS implants and Alpha AMS implants described by the percentage of functioning devices vs time. (a) Clinical (black) and estimated laboratory (grey) reliability of the Alpha IMS. (b) Clinical (black) and estimated laboratory (grey) reliability of the Alpha AMS. The laboratory reliability of the implant has been calculated according to the method described by Daschner et al. ${ }^{(15)}$ The reliability of the Alpha AMS implant is considerably higher than that of the Alpha IMS implant. 


\section{Results}

\subsection{Chip functionality}

\subsubsection{Electrical functionality}

The chip operation was verified to exhibit the following key features for optimized lifetime. A differential stimulation output voltage step of $2.4 \mathrm{~V}$ (from -1.2 to $+1.2 \mathrm{~V}$ ) is achieved, although the total maximum external voltages supplying the chip do not exceed $\pm 1.2 \mathrm{~V}$. That means that no voltage overhead is required for precise chip operation. All terminals, including the supply, control inputs, and electrodes, are close to DC-free. No silicon chip working under these conditions has been reported so far, to the best of our knowledge. The electrodes are driven with a three-phase current waveform, negative precharge (down to $-1.2 \mathrm{~V}$ maximum), positive stimulating charge, and negative discharge (for perfect charge balancing). The resulting voltage waveform is close to DC-free, resulting in the longest lifetime for the electrodes. To further increase the lifetime, the supply voltage alternation frequency can be adjusted to be significantly higher than the stimulation repetition rate.

\subsubsection{Charge transfer characteristic}

In Fig. 4, a typical characteristic charge transfer curve of the Alpha AMS implant is shown. At a fixed sensitivity, light levels ranging across approximately $1.5 \log$ units are converted to charge pulses by each pixel with a sigmoidal relationship. Increasing the sensitivity shifts the curve by several log units to lower light levels. For the maximum dynamic range of visual perception, the light conditions must be in the rising edge of the characteristic charge transfer curve. For higher sensitivity values, already low light levels result in a large stimulation signal. Increasing the gain increases the maximum amplitude of the stimulation signal and thus the released charge per pixel. The necessary gain for each patient depends mainly on the excitability of the retina of the patient and has to be adjusted for each patient. For an electrode with a diameter of $30 \mu \mathrm{m}$, the maximum charge per electrode shown in Fig. 4(a) corresponds to a charge density of about $1.7 \mathrm{mC} / \mathrm{cm}^{2}$.

\subsection{Reliability of the subretinal implant}

\subsubsection{Results of laboratory accelerated aging tests of the CMOS chip}

In Fig. 5, the results of laboratory aging tests of the Alpha IMS and the Alpha AMS CMOS chip are shown. The reliability curve describes the percentage of devices that are expected to still function after a particular length of time. For both datasets, a Weibull distribution model was used for a quantitative analysis. The reliability of the Alpha AMS CMOS chip is considerably higher than that of the Alpha IMS CMOS chip. For the Alpha IMS, the Weibull distribution of the CMOS chip is almost at the limit of an exponential distribution with a median 
lifetime of 14.6 months. This is in accordance with the fact that more than half of the observed failure modes during the clinical trial of the Alpha IMS were corrosion of the CMOS chip. For the Alpha AMS CMOS chip, the laboratory data show a Weibull distribution with a median lifetime of 72.5 months. In the clinical trial of the Alpha AMS, the maximum time for which an implant was implanted and still remained functional is 40 months. The observation time of the implants during the clinical trial is therefore still much shorter than the expected lifetime of the Alpha AMS CMOS chip. The longer lifetime of the Alpha AMS CMOS chip is supported by the fact that no failures due to the degradation of the CMOS chip have yet been observed in the clinical use of the Alpha AMS.

\subsubsection{Lifetime of implanted devices}

The reliability of medical devices is usually illustrated using a Kaplan-Meier plot. The cumulative survival rate in this case means how many of the implants are expected to function until a specific time. Owing to one reimplantation of an Alpha AMS device in one patient during the clinical trial, the 16 study patients correspond to 17 implants. Also for the calculation of the reliability of the Alpha AMS implant, in addition to the 16 patients in the clinical trials, 4 patients that are not part of a clinical trial have been included. Therefore, all calculations of the reliability of the implants are based on 21 Alpha AMS implant devices. In Fig. 6, the cumulative survival rate is illustrated.

All Alpha IMS implants have failed until now. In contrast, as of 06/30/2017, the maximum implanted time of an Alpha AMS device is 40 months, and 14 out of the 21 implanted Alpha AMS devices are still functioning. While for the Alpha IMS only $32 \%$ of the implants were still functioning after the first year, the cumulative survival rate of the Alpha AMS was $81 \%$ after one year, even when all types of failure modes are included $(\mathrm{C} 1+\mathrm{C} 2)$. Considering only technical failures (category $\mathrm{C} 1$ ), the cumulative survival rate was $95 \%$ after one year. The reason for the high failure rate of the Alpha IMS implant was technical failure of the CMOS chip itself in most cases. ${ }^{(15)}$ Improvements in the chip encapsulation and voltage management led to a more robust implant. ${ }^{(7)}$ So far, none of the technical failures of the Alpha AMS implant have been related to a failure of the conformal coating.

\subsubsection{Clinical and laboratory reliability}

In Fig. 7, the reliability calculated by laboratory accelerated aging tests of the implants Alpha IMS [Fig. 7(a)] and Alpha AMS [Fig. 7(b)] is compared with the clinical reliability of the two devices. The laboratory reliability has been calculated by multiplication of the reliability of each of the implant components, as described in detail by Daschner et al. ${ }^{(15)}$ Two-sided $75 \%$ confidence levels are included for the clinical data. For the Alpha IMS [Fig. 7(a)], good agreement between the observed clinical lifetime and the laboratory calculation is observed. The Alpha AMS shows a considerably longer lifetime than the Alpha IMS in the clinical data as well as in the laboratory data. The estimated reliability from the laboratory data is slightly higher than the reliability observed in clinical use because in laboratory experiments, only 
technical failures can be modeled, while in clinical use, other failure modes, such as procedural errors, can also occur. Fourteen of the 21 Alpha AMS implants in clinical use are still functioning. Therefore, an estimate of the clinical lifetime can only be based on the failures that occurred before 06/30/2017. However, the current average runtime of the Alpha AMS implants is already longer than the lifetime of the Alpha IMS implants.

\subsection{Clinical performance}

Implant-mediated visual perception was observed in 13 out of 15 patients that participated in the clinical trials. ${ }^{(7)}$ Two patients were able to distinguish Landolt C-rings of 20/1111 and 20/546. Twelve patients could achieve a basic grating acuity between 0.1 and $3.3 \mathrm{cpd}$. Detection, localization, and counting of objects was significantly better with the implant "ON" than with the implant "OFF" over the whole observation period. On average, $4.6 \pm 0.8$ of 6 different grey levels could be distinguished. The visual perception with the Alpha AMS was stable over the observation period of 12 months after surgery.

\section{Discussion}

The functionality of subretinal implants depends heavily on the performance of the chip that establishes the electronic interface to the retina. The chip of the Alpha AMS samples the retinal image similarly to a camera chip and stimulates the adjacent retina simultaneously at multiple locations.

From the characteristic charge transfer curve recorded from a retinal chip under defined illumination, the region of maximum light sensitivity can be shifted through the whole range of relevant illumination conditions. Since the dynamic range of the photodiodes covers the whole range of ambient light intensity levels, the sensitivity can be adjusted from $10^{4} \mathrm{~lx}$, which is typical for extremely bright scenes in direct sunlight, to below $10^{-2} 1 \mathrm{x}$, which is sufficient to see objects under street light illumination at night. ${ }^{(20)}$ The stimulation strength can be adjusted to match the necessary stimulating current to the individual patient's needs, depending on the degradation level of the patient's retina.

Several material and design changes ${ }^{(7)}$ led to a significant improvement of the durability of the current implant Alpha AMS compared with the predecessor Alpha IMS. From the KaplanMeier plot, which shows the current status of the functionality of all implants in clinical use (Fig. 6), it can be seen that the runtime of the Alpha AMS implant is already significantly longer than that of the Alpha IMS implant. The most common failure mode of the Alpha IMS implant, which was the CMOS chip itself, has been eliminated. Considering the CMOS chip alone, the measured median lifetime in laboratory experiments has been increased from 14.6 months (Alpha IMS) to 72.5 months (Alpha AMS), corresponding to a factor of 5.0. ${ }^{(15)}$ By fitting the observed clinical lifetimes with an exponential model (Fig. 7), a considerably longer functional lifetime is estimated for the Alpha AMS that is slightly smaller than the distribution determined from laboratory experiments owing to the fact that, in the laboratory, only purely technical failures can be considered. 
The lifetime of the implant Alpha IMS devices in clinical use has been estimated very precisely with the statistical model used for analyzing the accelerated aging tests [Fig. 7(a)]. Using the same method and the model, validated with the clinical data of the Alpha IMS device, we anticipate a median clinical lifetime of the Alpha AMS device of almost 5 years. ${ }^{(15)}$

Implant-mediated light perception has been demonstrated in clinical trials. Comparison with other commercially available retinal implants shows that, to date, the Alpha AMS has the highest theoretical resolution as well as the highest measured visual acuity in clinical use. ${ }^{(1)}$ In clinical trials with both the Alpha IMS and the Alpha AMS, a visual acuity of 20/546 has been achieved. ${ }^{(7,21)}$ The reason why the theoretical visual acuity has not been reproduced in clinical use so far may be attributed to the fact that each electrode can activate cells in a region with a radius of more than $100 \mu \mathrm{m}$ around the stimulating electrode. Therefore, the separate perception of simultaneously activated neighboring pixels might be difficult.

\section{Conclusions}

The current subretinal implant, Alpha AMS, and its predecessor, Alpha IMS, proved to be effective and safe in previous studies. ${ }^{(8,22)}$ In comparison with the Alpha IMS, the Alpha AMS achieves similar visual results ${ }^{(7)}$ with an extended lifetime. ${ }^{(15)}$

The Alpha AMS is a microsized implant specially designed to maximize visual sensations by using a stimulation chip that measures the local light intensity at each stimulation location, instead of using an external camera, and which is not hermetically encapsulated. The theoretical model used to predict the clinical reliability of this unique neurotechnological device has been validated by comparing the actual lifetime of the Alpha IMS implants during clinical use with the theoretically calculated lifetime from laboratory accelerated aging tests. Therefore, it is expected that for the Alpha AMS, the lifetime in clinical use will also match the lifetime determined by laboratory experiments. This is supported by the observation that over a period of one year in the clinical trial, implant-mediated light perception was stable.

\section{References}

1 A. T. Chuang, C. E. Margo, and P. B. Greenberg: Br. J. Ophthalmol. 98 (2014) 852.

2 M. Gerhardt, J. Alderman, and A. Stett: IEEE Trans. Neural Syst. Rehabil. Eng. 18 (2010) 1.

3 A. Stett, A. Mai, and T. Herrmann: J. Neural Eng. 4 (2007) 7.

4 M. Eickenscheidt, M. Jenkner, R. Thewes, P. Fromherz, and G. Zeck: J. Neurophysiol. 107 (2012) 2742.

5 R. E. MacLaren: Eye (London) 31 (2017) 191.

6 E. Zrenner, K. U. Bartz-Schmidt, D. Besch, F. Gekeler, A. Koitschev, H. G. Sachs, and K. Stingl: The Subretinal Implant Alpha: Implantation and Functional Results: In Artificial Vision (Springer International Publishing., Munich, 2017) p. 65.

7 K. Stingl, R. Schippert, K. U. Bartz-Schmidt, D. Besch, C. L. Cottriall, T. L. Edwards, F. Gekeler, U. Greppmaier, K. Kiel, A. Koitschev, L. Kühlewein, R. E. MacLaren, J. D. Ramsden, J. Roider, A. Rothermel, H. Sachs, G. S. Schröder, J. Tode, N. Troelenberg, and E. Zrenner: Front. Neurosci. 11 (2017) 445.

8 K. Stingl, K. U. Bartz-Schmidt, D. Besch, C. K. Chee, C. L. Cottriall, F. Gekeler, M. Groppe, T. L. Jackson, R. E. MacLaren, A. Koitschev, A. Kusnyerik, J. Neffendorf, J. Nemeth, M. A. Naeem, T. Peters, J. D. Ramsden, H. Sachs, A. Simpson, M. S. Singh, B. Wilhelm, D. Wong, and E. Zrenner: Vision Res. 111 (2015) 149.

9 E. Zrenner, K. U. Bartz-Schmidt, H. Benav, D. Besch, A. Bruckmann, V. P. Gabel, F. Gekeler, U. Greppmaier, A. Harscher, S. Kibbel, J. Koch, A. Kusnyerik, T. Peters, K. Stingl, H. Sachs, A. Stett, P. Szurman, B. Wilhelm, and R. Wilke: Proc. R. Soc. B 278 (2011) 1489. 
10 A. Rothermel, L. Liu, N. P. Aryan, M. Fischer, J. Wuenschmann, S. Kibbel, and A. Harscher: IEEE J. SolidState Circuits 44 (2009) 290.

11 S. Negi, R. Bhandari, and F. Solzbacher: J. Sens. Technol. 2 (2012) 138.

12 J. D. Weiland, D. J. Anderson, and M. S. Humayun: IEEE Trans. Biomed. Eng. 49 (2002) 1574.

13 M. Katz and P. B. Kruger: The Human Eye as an Optical System: In Duane's Ophthalmology on CD-ROM (Lippincott Williams \& Wilkins, Philadelphia, 2006).

14 H. Wilker: Weibull-Statistik in der Praxis. Leitfaden zur Zuverlässigkeitsermittlung Technischer Komponenten (Books on Demand, 2004).

15 R. Daschner, U. Greppmaier, M. Kokelmann, S. Rudorf, R. Rudorf, S. Schleehauf, and W. G. Wrobel: Biomed. Microdevices 19 (2017) 7.

16 K. J. Hemmerich: Med. Plast. Biomater. 5 (1998) 16.

17 E. L. Kaplan and P. Meier: J. Amer. Statistical Assoc. 53 (1958) 457.

18 K. Stingl, K. U. Bartz-Schmidt, A. Braun, F. Gekeler, U. Greppmaier, A. Schatz, A. Stett, T. Strasser, V. Kitiratschky, and E. Zrenner: Doc. Ophthalmol. 133 (2016) 81.

19 R. Wilke, M. Bach, B. Wilhelm, W. Durst, S. Trauzettel-Klosinski, and E. Zrenner: Testing Visual Functions in Patients with Visual Prostheses: In Artificial Sight. Biological and Medical Physics, Biomedical Engineering (Springer, New York, 2007) p. 91.

20 N. Pour-Aryan and A. Rothermel: 1st International Conference on Photonics, Optics and Laser Technology (PHOTOPTICS 2013) (2013).

21 K. Stingl, K. U. Bartz-Schmidt, D. Besch, A. Braun, A. Bruckmann, F. Gekeler, U. Greppmaier, S. Hipp, G. Hörtdörfer, C. Kernstock, A. Koitschev, A. Kusnyerik, H. Sachs, A. Schatz, K. T. Stingl, T. Peters, B. Wilhelm, and E. Zrenner: Proc. R. Soc. B 280 (2013) 20130077.

22 E. Zrenner, K. Bartz-Schmidt, T. Edwards, R. MacLaren, J. Roider, H. Sachs, and K. Stingl: 16th EURETINA Congress, Copenhagen (2016), http://bit.ly/2BTdtFv 